\title{
LLL-REDUCTION FOR INTEGER KNAPSACKS
}

\author{
ISKANDER ALIEV AND MARTIN HENK
}

\begin{abstract}
Given a matrix $A \in \mathbb{Z}^{m \times n}$ satisfying certain regularity assumptions, a well-known integer programming problem asks to find an integer point in the associated knapsack polytope

$$
P(A, \boldsymbol{b})=\left\{\boldsymbol{x} \in \mathbb{R}_{\geq 0}^{n}: A \boldsymbol{x}=\boldsymbol{b}\right\}
$$

or determine that no such point exists. We obtain an LLL-based polynomial time algorithm that solves the problem subject to a constraint on the location of the vector $\boldsymbol{b}$.
\end{abstract}

\section{Introduction and Statement of Results}

Let $A \in \mathbb{Z}^{m \times n}, 1 \leq m<n$, be an integral $m \times n$ matrix satisfying

i) $\operatorname{gcd}\left(\operatorname{det}\left(A_{I_{m}}\right): A_{I_{m}}\right.$ is an $m \times m$ minor of $\left.A\right)=1$,

ii) $\left\{\boldsymbol{x} \in \mathbb{R}_{\geq 0}^{n}: A \boldsymbol{x}=\mathbf{0}\right\}=\{\mathbf{0}\}$,

where $\operatorname{gcd}\left(a_{1}, \ldots, a_{l}\right)$ denotes the greatest common divisor of integers $a_{i}$, $1 \leq i \leq l$. For such a matrix $A$ and a vector $\boldsymbol{b} \in \mathbb{Z}^{m}$ the knapsack polytope $P(A, \boldsymbol{b})$ is defined as

$$
P(A, \boldsymbol{b})=\left\{\boldsymbol{x} \in \mathbb{R}_{\geq 0}^{n}: A \boldsymbol{x}=\boldsymbol{b}\right\} .
$$

Observe that on account of (1.1) ii), $P(A, \boldsymbol{b})$ is indeed a polytope (or empty).

The paper is concerned with the following integer programming problem:

Given input $(A, \boldsymbol{b})$, find an integer point in $P(A, \boldsymbol{b})$

or determine that no such point exists .

The problem (1.2) is NP-hard (see e.g. Section 15.6 in Papadimitriou and Steiglitz [20]). When $m=1$ we obtain the well-known integer knapsack problem: given integers $a_{j}, j=1, \ldots, n$, and $b$, find integers $x_{j} \geq 0, j=$ $1, \ldots, n$, such that $\sum_{j=1}^{n} a_{j} x_{j}=b$ or determine that no such integers exist.

Let us define the set

$$
\mathcal{F}(A)=\left\{\boldsymbol{b} \in \mathbb{Z}^{m}: P(A, \boldsymbol{b}) \cap \mathbb{Z}^{n} \neq \emptyset\right\} .
$$

Thus, the set $\mathcal{F}(A)$ will consist of all possible vectors $\boldsymbol{b}$ such that the polytope $P(A, \boldsymbol{b})$ contains an integer point.

2000 Mathematics Subject Classification. Primary: 90C10, 90C27, 11D07 ; Secondary: $11 \mathrm{H} 06$.

Key words and phrases. Knapsack problem; Frobenius numbers; successive minima; inhomogeneous minimum; distribution of lattices. 
A set $S \subset \mathbb{R}^{m}$ will be called a feasible set if $S \cap \mathbb{Z}^{m} \subset \mathcal{F}(A)$. Results of Aliev and Henk 2], Knight [15], Simpson and Tijdeman [26] and Pleasants, Ray and Simpson 21] show that the set $\mathcal{F}(A)$ can be decomposed into the set of all integer points in a certain feasible (translated) cone and a complementary set with complex combinatorial structure.

Note that the case $m=1$ corresponds to the celebrated Frobenius problem and has been extensively studied in the literature. We address this problem below. When $n=m+1$ Pleasants, Ray and Simpson 21] obtained a unique maximal cone whose interior is feasible. To the best of the authors knowledge the existence of such a maximal cone in the general case is not known.

The location of a feasible cone is given by the diagonal Frobenius number defined as follows. Let $\boldsymbol{v}_{1}, \ldots, \boldsymbol{v}_{n} \in \mathbb{Z}^{m}$ be the columns of the matrix $A$ and let

$$
C=\left\{\mu_{1} \boldsymbol{v}_{1}+\cdots+\mu_{n} \boldsymbol{v}_{n}: \mu_{1}, \ldots, \mu_{n} \geq 0\right\}
$$

be the cone generated by $\boldsymbol{v}_{1}, \ldots, \boldsymbol{v}_{n}$. Let also $\boldsymbol{v}:=\boldsymbol{v}_{1}+\ldots+\boldsymbol{v}_{n}$. Following Aliev and Henk [2, by the diagonal Frobenius number $\mathrm{g}=\mathrm{g}(A)$ of $A$ we understand the minimal $s \geq 0$, such that for all $\boldsymbol{b} \in(s \boldsymbol{v}+C) \cap \mathbb{Z}^{m}$ the polytope $P(A, \boldsymbol{b})$ contains an integer point. Thus we have the inclusion

$$
(\mathrm{g}(A) \boldsymbol{v}+C) \cap \mathbb{Z}^{m} \subset \mathcal{F}(A),
$$

or, in other words, the translated cone $\mathrm{g}(A) \boldsymbol{v}+C$ is feasible.

The behavior of $\mathrm{g}(A)$ was investigated in Aliev and Henk 2. The authors obtained an optimal up to a constant multiplier upper bound

$$
\mathrm{g}(A) \leq \frac{(n-m)}{2}\left(n \operatorname{det}\left(A A^{T}\right)\right)^{1 / 2}
$$

and estimated the expected value of the diagonal Frobenius number.

It is natural to expect that the problem (1.2) is solvable in polynomial time when the right hand side vector $\boldsymbol{b}$ belongs to a feasible cone. For such vectors $\boldsymbol{b}$ we a priori know that the knapsack polytope contains at least one integer point. We would like to propose the following conjecture.

Conjecture 1.1. The problem (1.2) is solvable in polynomial time for all instances $(A, \boldsymbol{b})$ with

$$
\boldsymbol{b} \in(\mathrm{g}(A) \boldsymbol{v}+C) \cap \mathbb{Z}^{m}
$$

This question is closely related to algorithmic problems in Section A.1 of Ramírez Alfonsín [22].

The first result of the paper gives an estimate for the location of the desired feasible cone and can be considered as a step towards proving our conjecture.

Theorem 1.1. There exists a polynomial time algorithm which, given $(A, \boldsymbol{b})$, where A satisfies (1.1), $\boldsymbol{b} \in \mathbb{Z}^{m}$ with

$$
\boldsymbol{b} \in\left(2^{(n-m) / 2-1} p(m, n)\left(\operatorname{det}\left(A A^{T}\right)\right)^{1 / 2} \boldsymbol{v}+C\right)
$$


and

$$
p(m, n)=2^{-1 / 2}(n-m)^{1 / 2} n^{1 / 2},
$$

finds an integer point in the polytope $P(A, \boldsymbol{b})$.

In view of (1.3), the affirmative answer to our conjecture would imply that the factor $2^{(n-m) / 2-1} p(m, n)$ in (1.4) can be replaced by $\frac{(n-m) n^{1 / 2}}{2}$, hence the exponent $2^{(n-m) / 2-1}$ in (1.4) might be redundant.

Our next result shows that the exponent can be removed for all matrices $A$ with sufficiently large $\operatorname{det}\left(A A^{T}\right)$. This phenomenon is related to the bounds on the efficiency of the LLL-algorithm and is a consequence of Theorem 1.4 below. In order to state the result, let $\gamma_{k}$ be the $k$-dimensional Hermite constant for which we refer to [18, Definition 2.2.5]. Here we just note that by a result of Blichfeldt (see, e.g., Gruber and Lekkerkerker [11])

$$
\gamma_{k} \leq 2\left(\frac{k+2}{\sigma_{k}}\right)^{2 / k},
$$

where $\sigma_{k}$ is the volume of the unit $k$-ball; thus $\gamma_{k}=O(k)$.

Theorem 1.2. There exists a polynomial time algorithm which, given $(A, \boldsymbol{b})$, where A satisfies (1.1), $\boldsymbol{b} \in \mathbb{Z}^{m}$ with

$$
\boldsymbol{b} \in\left(p(m, n)\left(\operatorname{det}\left(A A^{T}\right)\right)^{1 / 2} \boldsymbol{v}+C\right)
$$

and

$$
\operatorname{det}\left(A A^{T}\right)>\frac{(n-m) 2^{2(n-m-2)} \gamma_{n-m}^{n-m}}{n^{2}},
$$

finds an integer point in the polytope $P(A, \boldsymbol{b})$.

Thus, if the dimension $n$ is concerned, Theorem 1.1 gives an exponential bound in $n$ for the location of the desired feasible cone, the affirmative answer to Conjecture 1.1 would imply the bound of order $n^{3 / 2}$ and for large determinants $\operatorname{det}\left(A A^{T}\right)$ we obtained the bound of order $n$ in Theorem 1.2, In view of the size of $\gamma_{k}$, the lower bound for $\operatorname{det}\left(A A^{T}\right)$ in (1.6) has order $n^{-1} 2^{n \log n+2 n}$.

We would also like to mention an interesting consequence of Theorems 1.1 and 1.2. The proof of Lemma 1.1 in Aliev and Henk 22 immediately implies that for any integer vector $\boldsymbol{w}$ in the interior int $C$ of the cone $C$ we have

$$
\left(\frac{\operatorname{det}\left(A A^{T}\right)}{n-m+1}\right)^{1 / 2} \boldsymbol{w} \in(\boldsymbol{v}+C) .
$$

It follows then from Theorem 1.1 that for every integer vector $\boldsymbol{b} \in \operatorname{int} C$ one can find in polynomial time an integer point in the polytope $P(A, \gamma \boldsymbol{b})$ for any integer vector $\gamma \boldsymbol{b}$ with

$$
\gamma>\frac{2^{(n-m) / 2-1} p(m, n)}{n-m+1} \operatorname{det}\left(A A^{T}\right) .
$$


Moreover, if we assume (1.6) to hold, then by Theorem 1.2 we can remove the exponential multiplier $2^{(n-m) / 2-1}$ from the latter inequality.

Let us now consider the special case $m=1$. Then $A=\boldsymbol{a}^{T}$ with $\boldsymbol{a}=$ $\left(a_{1}, a_{2}, \ldots, a_{n}\right)^{T} \in \mathbb{Z}^{n}$ and (1.1) i) says that $\operatorname{gcd}(\boldsymbol{a}):=\operatorname{gcd}\left(a_{1}, a_{2}, \ldots, a_{n}\right)=$ 1. Due to the second assumption (1.1) ii) we may assume that all entries of $\boldsymbol{a}$ are positive. The largest integral value $b$ such that for $A=\boldsymbol{a}^{T}$ and $\boldsymbol{b}=(b)$ the polytope $P(A, \boldsymbol{b})$ contains no integer point is called the Frobenius number of $\boldsymbol{a}$, denoted by $\mathrm{F}(\boldsymbol{a})$. Frobenius numbers naturally appear in the analysis of integer programming algorithms (see, e.g., Aardal and Lenstra [1, Hansen and Ryan [12], and Lee, Onn and Weismantel [16]). The general problem of finding $\mathrm{F}(\boldsymbol{a})$ has been traditionally referred to as the Frobenius problem. This problem is NP-hard (Ramírez Alfonsín [23, 22]) and integer programming techniques are known to be an effective tool for investigating behavior of the Frobenius numbers, see e.g. Kannan [13, Eisenbrand and Shmonin [7] and Beihoffer et al [5].

Thus, when $m=1$ the answer for the feasibility problem

Given input $(A, \boldsymbol{b})$, does the polytope $P(A, \boldsymbol{b})$ contain an integer point?

is affirmative for all instances $\left(\boldsymbol{a}^{T}, b\right)$ with $b>\mathrm{F}(\boldsymbol{a})$. Therefore, it is natural to expect that for $m=1$ the problem (1.2) can be solved in polynomial time when $b>c$, for some function $c=c(\boldsymbol{a})$. To the best of our knowledge, this conjecture with $c=\mathrm{F}(\boldsymbol{a})$ was first stated by Ramírez Alfonsín (for related algorithmic questions see Section A.1 in [22]). Note that if the answer to the latter conjecture is affirmative, then the factor $2^{(n-1) / 2-1} p(1, n)$ in (1.4) can be replaced by an absolute constant.

Let $\|\cdot\|$ denote the Euclidean norm. In the case $m=1$, Theorems 1.1 and 1.2 deal with input instances $\left(\boldsymbol{a}^{T}, b\right)$, satisfying the inequalities $b>2^{(n-1) / 2-1} p(1, n)\|\boldsymbol{a}\| \sum_{i=1}^{n} a_{i}$ and $b>p(1, n)\|\boldsymbol{a}\| \sum_{i=1}^{n} a_{i}$, respectively. However, in this important special case, one can use slightly refined lower bounds for $b$. The bounds naturally follow from the geometric approach to the Frobenius problem developed in Kannan [13] and are closely related to the upper bound obtained in Fukshansky and Robins [8].

Let $\boldsymbol{a}[i]=\left(a_{1}, \ldots, a_{i-1}, a_{i+1}, \ldots, a_{N}\right)$. For $m=1$ we obtain the following refinement of Theorems 1.1 and 1.2 .

Theorem 1.3. Let $\delta>0$. Then the conditions (1.4) and (1.5) in the statements of Theorems 1.1] and 1.2 can be replaced by

$$
b \geq 2^{(n-1) / 2-1}(1+\delta) p(1, n) \sum_{i=1}^{n}\|\boldsymbol{a}[i]\| a_{i}
$$

and

$$
b \geq(1+\delta) p(1, n) \sum_{i=1}^{n}\|\boldsymbol{a}[i]\| a_{i},
$$


respectively.

The proofs of Theorems 1.1 and 1.2 are based on the classical Babai's nearest point algorithm [4. The algorithm is searching for a nearby lattice point and is built on the LLL lattice basis reduction (see Section 3). The key ingredient of the proof of Theorem 1.2 is the following result.

Theorem 1.4. Let $\rho_{k}=\frac{k 2^{2(k-2) \gamma_{k}^{k}}}{n^{2}}$. If $L \subset \mathbb{Z}^{n}$ is a $k$-dimensional lattice and $\boldsymbol{b}_{1}, \boldsymbol{b}_{2}, \ldots, \boldsymbol{b}_{k}$ is an $L L L$-reduced basis of $L$, then

$$
\left\|\boldsymbol{b}_{i}\right\| \leq\left(1+\frac{\rho_{k}}{(\operatorname{det}(L))^{2}}\right)^{1 / 2} \sqrt{n} \operatorname{det}(L), \quad i=1, \ldots, k .
$$

Note that the classical bounds for the lengths of the vectors in an LLLreduced basis imply for all $1 \leq i \leq k$ the estimates

$$
\left\|\boldsymbol{b}_{i}\right\| \leq 2^{\frac{k-1}{2}} n^{1 / 2} \operatorname{det}(L),
$$

see Lemma 3.3 below. In (1.10) we manage to remove the exponential multiplier $2^{(k-1) / 2}$ for integer lattices with sufficiently large determinant.

\section{Integer Knapsacks and Geometry of Numbers}

Our approach to the problem is based on Geometry of Numbers for which we refer to the books [6, 10, 11].

By a lattice we will understand a discrete submodule $L$ of a finite-dimensional Euclidean space. Here we are mainly interested in primitive lattices $L \subset \mathbb{Z}^{n}$, where such a lattice is called primitive if $L=\operatorname{span}_{\mathbb{R}}(L) \cap \mathbb{Z}^{n}$.

Recall that the Frobenius number $\mathrm{F}(\boldsymbol{a})$ is defined only for integer vectors $\boldsymbol{a}=\left(a_{1}, a_{2}, \ldots, a_{n}\right)$ with $\operatorname{gcd}(\boldsymbol{a})=1$. This is equivalent to the statement that the 1-dimensional lattice $L=\mathbb{Z} \boldsymbol{a}$, generated by $\boldsymbol{a}$ is primitive. This generalizes easily to an $m$-dimensional lattice $L \subset \mathbb{Z}^{n}$ generated by $\boldsymbol{a}_{1}, \ldots, \boldsymbol{a}_{m} \in \mathbb{Z}^{n}$. Here the criterion is that $L$ is primitive if and only if the greatest common divisor of all $m \times m$-minors is 1 . This is an immediate consequence of Cassels [6, Lemma 2, Chapter1] or see Schrijver [25, Corollary 4.1c].

Hence, by our assumption (1.1) i), the rows of the matrix $A$ generate a primitive lattice $L_{A}$. The determinant of an $m$-dimensional lattice is the $m$ dimensional volume of the parallelepiped spanned by the vectors of a basis. Thus in our setting we have

$$
\operatorname{det}\left(L_{A}\right)=\sqrt{\operatorname{det}\left(A A^{T}\right)} .
$$

Now let $A \in \mathbb{Z}^{m \times n}$ be a matrix satisfying the assumptions (1.1). By $V_{A}$ we will denote the $m$-dimensional subspace of $\mathbb{R}^{n}$ spanned by the rows of $A$. The orthogonal complement of $V_{A}$ in $\mathbb{R}^{n}$ will be denoted as $V_{A}^{\perp}$, so that

$$
V_{A}^{\perp}=\left\{\boldsymbol{x} \in \mathbb{R}^{n}: A \boldsymbol{x}=\mathbf{0}\right\} .
$$


Furthermore, we will use the notation

$$
L_{A}^{\perp}=V_{A}^{\perp} \cap \mathbb{Z}^{n}
$$

for the integer sublattice contained in $V_{A}^{\perp}$. Observe that (see e. g. [19])

$$
\operatorname{det}\left(L_{A}^{\perp}\right)=\operatorname{det}\left(L_{A}\right)=\sqrt{\operatorname{det}\left(A A^{T}\right)} .
$$

For a $k$-dimensional lattice $L$ and an 0 -symmetric convex body $K \subset$ $\operatorname{span}_{\mathbb{R}} L$ the $i$ th-successive minimum of $K$ with respect to $L$ is defined as

$$
\lambda_{i}(K, L)=\min \{\lambda>0: \operatorname{dim}(\lambda K \cap L) \geq i\}, \quad 1 \leq i \leq k,
$$

i.e., it is the smallest factor such that $\lambda K$ contains at least $i$ linearly independent lattice points of $L$.

The Minkowski's celebrated theorem on successive minima states (cf. [10, Theorem 23.1])

$$
\frac{2^{k}}{k !} \operatorname{det}(L) \leq \operatorname{vol}(K) \prod_{i=1}^{k} \lambda_{i}(K, L) \leq 2^{k} \operatorname{det}(L),
$$

where vol $(K)$ denotes the volume of $K$.

Let $B$ be the unit ball in $\operatorname{span}_{\mathbb{R}} L$. In the important special case $K=B$ the Minkowski's theorem on successive minima can be improved (cf. [11, $\S 18.4$, Theorem 3]) to

$$
\operatorname{det}(L) \leq \prod_{i=1}^{k} \lambda_{i}(B, L) \leq \gamma_{k}^{k / 2} \operatorname{det}(L) .
$$

\section{Auxiliary Results}

First, we will prove several lemmas that show a relation between the LLL reduction and successive minima.

For a basis $\boldsymbol{b}_{1}, \boldsymbol{b}_{2}, \ldots, \boldsymbol{b}_{k}$ of a lattice $L$ in $\mathbb{R}^{n}$ we denote by $\hat{\boldsymbol{b}}_{1}, \hat{\boldsymbol{b}}_{2}, \ldots, \hat{\boldsymbol{b}}_{k}$ its Gram-Schmidt orthogonalization and by $\mu_{i, j}$ the corresponding GramSchmidt coefficients, that is

$$
\hat{\boldsymbol{b}}_{1}=\boldsymbol{b}_{1}, \quad \hat{\boldsymbol{b}}_{i}=\boldsymbol{b}_{i}-\sum_{j=1}^{i-1} \mu_{i j} \hat{\boldsymbol{b}}_{i}, \quad 2 \leq i \leq k,
$$

and

$$
\mu_{i j}=\frac{\left\langle\boldsymbol{b}_{i}, \hat{\boldsymbol{b}}_{j}\right\rangle}{\left\|\hat{\boldsymbol{b}}_{j}\right\|^{2}} .
$$

Put $\lambda_{i}=\lambda(B, L)$, where $B$ is the unit ball in $\operatorname{span}_{\mathbb{R}} L$. Let us recall the following technical observation.

Lemma 3.1. We have

$$
\lambda_{i} \geq \min _{j=i, i+1, \ldots, k}\left\|\hat{\boldsymbol{b}}_{j}\right\|, \quad i=1,2, \ldots, k .
$$


Proof. The proof can be easily derived from the proof of Proposition 1.12 in [17.

Recall that a lattice basis $\boldsymbol{b}_{1}, \boldsymbol{b}_{2}, \ldots, \boldsymbol{b}_{k}$ is $L L L$-reduced if

(a) $\left|\mu_{i j}\right| \leq \frac{1}{2}$, for $1 \leq j<i \leq k$;

(b) $\frac{3}{4}\left\|\hat{\boldsymbol{b}}_{i-1}\right\|^{2} \leq\left\|\hat{\boldsymbol{b}}_{i}\right\|^{2}+\mu_{i-1}^{2}\left\|\hat{\boldsymbol{b}}_{i-1}\right\|^{2}$, for $2 \leq i \leq k$.

The next lemma gives well-known upper bounds for the length the $i$ th vector of the LLL-reduced basis.

Lemma 3.2. Suppose that the basis $\boldsymbol{b}_{1}, \boldsymbol{b}_{2}, \ldots, \boldsymbol{b}_{k}$ is $L L L$-reduced. Then for $1 \leq i \leq k$ the inequalities

$$
\begin{gathered}
\left\|\boldsymbol{b}_{i}\right\|^{2} \leq 2^{i-1}\left\|\hat{\boldsymbol{b}}_{i}\right\|^{2} \\
\left\|\boldsymbol{b}_{i}\right\|^{2} \leq 2^{k-1} \lambda_{i}^{2}
\end{gathered}
$$

hold.

Proof. The inequalities (3.1) and (3.2) can be easily derived from the proofs of Propositions 1.6 and 1.12 in [17], respectively.

The next result gives an upper bound for the lengths of the vectors in an LLL-reduced basis in terms of the determinant of the lattice. The bound is based on the classical estimates from Lenstra, Lenstra and Lovasz [17] and, consequently, involves the exponential multiplier $2^{(k-1) / 2}$.

Lemma 3.3. Let $L \subset \mathbb{Z}^{n}$ be given by an $L L L$-reduced basis $\boldsymbol{b}_{1}, \boldsymbol{b}_{2}, \ldots, \boldsymbol{b}_{k}$. Then

$$
\max _{i=1, \ldots, k}\left\|\boldsymbol{b}_{i}\right\| \leq 2^{\frac{k-1}{2}} n^{1 / 2} \operatorname{det}(L)
$$

Proof. By Proposition 1.12 of Lenstra, Lenstra and Lovasz [17] for any choice of linearly independent vectors $\boldsymbol{x}_{1}, \ldots, \boldsymbol{x}_{k} \in L$ the inequality

$$
\left\|\boldsymbol{b}_{i}\right\| \leq 2^{\frac{k-1}{2}} \max \left\{\left\|\boldsymbol{x}_{1}\right\|, \ldots,\left\|\boldsymbol{x}_{k}\right\|\right\}
$$

holds.

Put $C^{n}=[-1,1]^{n}$, i.e., $C^{n}$ is the $n$-dimensional cube of edge length 2 centered at the origin. By a well-known result of Vaaler [27], any $k$ dimensional section of the cube $C^{n}$ has $k$-volume at least $2^{k}$. In particular we have

$$
\operatorname{vol}_{k}\left(C^{n} \cap \operatorname{span}_{\mathbb{R}}(L)\right) \geq 2^{k} .
$$

Thus, by the Minkowski theorem on successive minima, applied to the section $C^{n} \cap \operatorname{span}_{\mathbb{R}}(L)$ and $L$, there exist linearly independent vectors $\boldsymbol{x}_{1}, \ldots, \boldsymbol{x}_{k} \in$ $L$ such that

$$
\left\|\boldsymbol{x}_{1}\right\|_{\infty} \cdots\left\|\boldsymbol{x}_{k}\right\|_{\infty} \leq \operatorname{det}(L),
$$

where $\|\cdot\|_{\infty}$ denotes the maximum norm. 
Since $\boldsymbol{x}_{i}$ are nontrivial integral vectors we have

$$
\max \left\{\left\|\boldsymbol{x}_{1}\right\|_{\infty}, \ldots,\left\|\boldsymbol{x}_{k}\right\|_{\infty}\right\} \leq \operatorname{det}(L) .
$$

Combining the latter inequality with (3.4) we obtain the inequality (3.3).

The last lemma of this section gives an upper bound for the last successive minimum in terms of the determinant of the lattice.

\section{Lemma 3.4.}

$$
\lambda_{k} \leq \sqrt{n} \operatorname{det}(L)
$$

Proof. By the Minkowski theorem on successive minima, applied to the set $C^{n} \cap \operatorname{span}_{\mathbb{R}}(L)$ and the lattice $L$, and by the already mentioned result of Vaaler [27], we have

$$
\prod_{i=1}^{k} \lambda_{i}\left(C^{n} \cap \operatorname{span}_{\mathbb{R}}(L), L\right) \leq \operatorname{det}(L) .
$$

Since $L \subset \mathbb{Z}^{n}$, the interior of $C^{n} \cap \operatorname{span}_{\mathbb{R}}(L)$ does not contain any nonzero point of $L$. This implies

$$
\lambda_{k}\left(C^{n} \cap \operatorname{span}_{\mathbb{R}}(L), L\right) \leq \operatorname{det}(L),
$$

so that

$$
\lambda_{k} \leq \sqrt{n} \operatorname{det}(L)
$$

\section{Proof of Theorem 1.4}

If for some $l<k$ we have $\left\|\boldsymbol{b}_{l}\right\|>\left\|\boldsymbol{b}_{k}\right\|$ then, similarly to the arguments below, it can be shown that the inequalities (1.10) hold. Thus we may assume that $\boldsymbol{b}_{k}$ is the longest vector of the basis $\boldsymbol{b}_{1}, \ldots, \boldsymbol{b}_{k}$. Now assume $\left\|\boldsymbol{b}_{k}\right\| \geq\left(1+c_{k}\right)^{1 / 2} \sqrt{n} \operatorname{det}(L)$ for some $c_{k}>0$. Write

$$
\boldsymbol{b}_{k}=\hat{\boldsymbol{b}}_{k}+\sum_{j<k} \mu_{k j} \hat{\boldsymbol{b}}_{j}, \quad\left|\mu_{k j}\right| \leq \frac{1}{2} .
$$

Hence

$$
\left\|\boldsymbol{b}_{k}\right\|^{2} \leq\left\|\hat{\boldsymbol{b}}_{k}\right\|^{2}+\frac{1}{4} \sum_{j<k}\left\|\hat{\boldsymbol{b}}_{j}\right\|^{2} .
$$

Since $\lambda_{k} \leq \sqrt{n} \operatorname{det}(L)$ by Lemma 3.4 and $\left\|\hat{\boldsymbol{b}}_{k}\right\|^{2} \leq \lambda_{k}^{2}$ by Lemma 3.1, one concludes

$$
\frac{1}{4} \sum_{j<k}\left\|\hat{\boldsymbol{b}}_{j}\right\|^{2} \geq c_{k} \cdot n \cdot(\operatorname{det}(L))^{2}
$$


Hence there is an $i \leq k-1$ with

$$
\left\|\boldsymbol{b}_{i}\right\|^{2} \geq 4 c_{k} \frac{n}{k}(\operatorname{det}(L))^{2} .
$$

For this $i$ one obtains by (3.2)

$$
\lambda_{i}^{2} \geq 4 c_{k} 2^{1-k} \frac{n}{k}(\operatorname{det}(L))^{2} .
$$

Using Lemma 3.1 and (3.1), one obtains $\lambda_{k}^{2} \geq\left\|\hat{\boldsymbol{b}}_{k}\right\|^{2} \geq 2^{1-k}\left\|\boldsymbol{b}_{k}\right\|^{2} \geq$ $2^{1-k}\left(1+c_{k}\right) n(\operatorname{det}(L))^{2} \geq 2^{1-k} n(\operatorname{det}(L))^{2}$. Therefore

$$
\prod_{j=1}^{k} \lambda_{j}^{2} \geq \lambda_{i}^{2} \lambda_{k}^{2} \geq c_{k} \frac{n^{2}}{k} 2^{2(2-k)}(\operatorname{det}(L))^{4} .
$$

Finally, Minkowski's second theorem (see (2.3) ) implies

$$
c_{k} \leq \frac{\rho_{k}}{(\operatorname{det}(L))^{2}} .
$$

\section{The Algorithm. Proofs of Theorems 1.1 and 1.2}

5.1. Proof of Theorem 1.1. We shall now give a high level description of an algorithm that satisfies conditions stated in Theorem 1.1. First the algorithm constructs an arbitrary integer solution $\boldsymbol{u}$ to $A \boldsymbol{x}=\boldsymbol{b}$ and a rational solution $\boldsymbol{c}$ to $A \boldsymbol{x}=\boldsymbol{b}$ with large positive coordinates. From this one computes an integer point $\boldsymbol{z}$ in $P(A, \boldsymbol{b})$ as follows. One finds a close vector $\boldsymbol{v}$ to $\boldsymbol{u}-\boldsymbol{c}$ in the lattice $L_{A}^{\perp}$ and considers $\boldsymbol{z}:=\boldsymbol{u}-\boldsymbol{v}$. The vector $\boldsymbol{z}$ is an integer vector, since $\boldsymbol{u}, \boldsymbol{v}$ are integer. It is a solution to $A \boldsymbol{x}=\boldsymbol{b}$, since $\boldsymbol{u}$ is and $A \boldsymbol{v}=0$. Next, observe that $\boldsymbol{z}=\boldsymbol{u}-\boldsymbol{v}=\boldsymbol{c}-(\boldsymbol{c}-\boldsymbol{u})-\boldsymbol{v}$, the vector $(\boldsymbol{c}-\boldsymbol{u})-\boldsymbol{v}$ is short, and $\boldsymbol{c}$ has large coordinates. This will imply $\boldsymbol{z} \in P(A, \boldsymbol{b})$.

Suppose that

$$
\boldsymbol{b} \in\left(\mu(m, n)\left(\operatorname{det}\left(A A^{T}\right)\right)^{1 / 2} \boldsymbol{v}+C\right) \cap \mathbb{Z}^{m}
$$

with $\mu(m, n)=2^{(n-m) / 2-1} p(m, n)$. The algorithm is presented below:

Input : $(A, \boldsymbol{b})$ with $A$ and $\boldsymbol{b}$ satisfying (1.1) and (5.1) respectively;

Output : $\boldsymbol{z} \in P(A, \boldsymbol{b}) \cap \mathbb{Z}^{n}$;

Step 1 : Find a basis $\boldsymbol{x}_{1}, \ldots, \boldsymbol{x}_{n-m}$ of $L_{A}^{\perp}$ and an integer solution $\boldsymbol{u}$ of the equation $A \boldsymbol{x}=\boldsymbol{b}$.

Step 2 : Find a point $\boldsymbol{c} \in P(A, \boldsymbol{b})$ with coordinates

$$
c_{i} \geq \mu(m, n)\left(\operatorname{det}\left(A A^{T}\right)\right)^{1 / 2}, \quad 1 \leq i \leq n .
$$

Step 3 : Apply the Babai's algorithm for finding a nearby lattice point to the basis $\boldsymbol{x}_{1}, \ldots, \boldsymbol{x}_{n-m}$ and the point $\boldsymbol{u}-\boldsymbol{c}$. The algorithm returns a lattice point $\boldsymbol{v} \in L_{A}^{\perp}$.

Step 4 : The output vector $\boldsymbol{z}:=\boldsymbol{u}-\boldsymbol{v}$. 
Let us now show that the algorithm satisfies conditions of Theorem 1.1.

Step 1 can be performed in polynomial time by Corollary 5.3c of Schrijver [25].

To justify Step 2 we will need the following observation.

Lemma 5.1. Let $\boldsymbol{b} \in(t \boldsymbol{v}+C) \cap \mathbb{Z}^{m}, t \geq 0$. One can find in polynomial time a point $\boldsymbol{c} \in P(A, \boldsymbol{b})$ with all coordinates $c_{i} \geq t$.

Proof. Since $\boldsymbol{b} \in(t \boldsymbol{v}+C)$, we have $\boldsymbol{b}=\sum_{i=1}^{n}\left(t+\delta_{i}\right) \boldsymbol{v}_{i}$ with $\delta_{i} \geq 0$. Therefore the polytope $P_{t}=\left\{\boldsymbol{x} \in P: x_{i} \geq t, 1 \leq i \leq n\right\}$ is not empty. By Lemma 6.5.1 of Grötschel, Lovász and Schrijver [9], one can find in polynomial time a vertex $\boldsymbol{c}$ of the polytope $P_{t}$. The point $c$ clearly satisfies conditions of Lemma 5.1

On account of (5.1), we can apply Lemma 5.1 with a rational number $t>\mu(m, n)\left(\operatorname{det}\left(A A^{T}\right)\right)^{1 / 2}$. Thus we obtain in polynomial time a point $\boldsymbol{c} \in P(A, \boldsymbol{b})$ with coordinates $c_{i}$ satisfying

$$
c_{i} \geq \mu(m, n)\left(\operatorname{det}\left(A A^{T}\right)\right)^{1 / 2}, \quad 1 \leq i \leq n .
$$

The algorithm of Babai (see 4]), applied at Step 3, runs in polynomial time as well. Thus it is enough to show that the output vector $\boldsymbol{z}$ is in the polytope $P(A, \boldsymbol{b})$.

Clearly, the polytope $P(A, \boldsymbol{b})$ contains a ball centered at $\boldsymbol{c}$ with radius $r \geq \min _{i} c_{i}$, so that

$$
r \geq \mu(m, n)\left(\operatorname{det}\left(A A^{T}\right)\right)^{1 / 2} .
$$

Since $A \boldsymbol{u}=\boldsymbol{b}$ and $\boldsymbol{v} \in L_{A}^{\perp}$, the output vector $\boldsymbol{z}$ satisfies the condition $A \boldsymbol{z}=\boldsymbol{b}$. Thus, by (5.2), it is enough to show that

$$
\|\boldsymbol{z}-\boldsymbol{c}\| \leq \mu(m, n)\left(\operatorname{det}\left(A A^{T}\right)\right)^{1 / 2} .
$$

The point $\boldsymbol{v}$, computed by Babai's algorithm, satisfies

$$
\|(\boldsymbol{u}-\boldsymbol{c})-\boldsymbol{v}\|^{2} \leq\left(\left\|\boldsymbol{b}_{1}\right\|^{2}+\cdots+\left\|\boldsymbol{b}_{n-m}\right\|^{2}\right) / 4,
$$

where $\boldsymbol{b}_{1}, \ldots, \boldsymbol{b}_{n-m}$ is an LLL-reduced basis of $L_{A}^{\perp}$. Since $\|\boldsymbol{z}-\boldsymbol{c}\|=\|(\boldsymbol{u}-$ $\boldsymbol{c}) \boldsymbol{- v} \|$, by (5.4) we have

$$
\|\boldsymbol{z}-\boldsymbol{c}\| \leq \frac{(n-m)^{1 / 2}}{2} \max _{i=1, \ldots, n-m}\left\|\boldsymbol{b}_{i}\right\| .
$$

By Lemma 3.3 and the choice of $\mu(m, n)$ we obtain the inequality (5.3).

5.2. Proof of Theorem 1.2, The above algorithm satisfies the statement of Theorem 1.2 as well. To see this, we only need to replace $\mu(m, n)=$ $2^{(n-m) / 2-1} p(m, n)$ by $\mu(m, n)=p(m, n)$ and to apply Theorem 1.4 instead of Lemma 3.3 in the end of the proof. 


\section{Case $m=1$. Proof of Theorem 1.3}

First we will show that the polytope $P\left(\boldsymbol{a}^{T}, b\right)$ contains a ball of sufficiently large radius whose center can be computed in polynomial time.

Lemma 6.1. The polytope $P\left(\boldsymbol{a}^{T}, b\right)$ contains an $(n-m)$-dimensional ball centered at a rational point $\boldsymbol{c}$ and of radius

$$
r>\frac{b\|\boldsymbol{a}\|}{(1+\delta) \sum_{i=1}^{n}\|\boldsymbol{a}[i]\| a_{i}} .
$$

The point $\boldsymbol{c}$ can be computed in polynomial time.

Proof. The polytope $P\left(\boldsymbol{a}^{T}, b\right)$ is the simplex with vertices $\boldsymbol{v}_{i}=\left(b / a_{i}\right) \boldsymbol{e}_{i}, 1 \leq$ $i \leq n$, where $\boldsymbol{e}_{i}$ are the standard basis vectors. Hence the inner unit normal vectors of the facets of this simplex (in the hyperplane $\left\{\boldsymbol{x} \in \mathbb{R}^{n}: \boldsymbol{a}^{T} \boldsymbol{x}=0\right\}$ ) are given by

$$
\boldsymbol{u}_{j}:=\frac{\|\boldsymbol{a}\|}{\|\boldsymbol{a}[j]\|}\left(\boldsymbol{e}_{j}-\frac{a_{j}}{\|\boldsymbol{a}\|^{2}} \boldsymbol{a}\right), \quad 1 \leq j \leq n .
$$

Here $\boldsymbol{e}_{j}$ denotes $j$-th unit vector in $\mathbb{R}^{n}$, and the facet corresponding to $\boldsymbol{u}_{j}$ is the convex hull of all vertices except $\left(b / a_{j}\right) \boldsymbol{e}_{j}$.

Now let $c^{*}$ be the center of the maximal inscribed ball in the simplex $P\left(\boldsymbol{a}^{T}, b\right)$, and let $r^{*}$ be its radius. Since this maximal ball touches all facets of the simplex, the radius is $(n-1)$ times the ratio of volume to surface area. Standard calculations (see, e.g., Fukshansky and Robins [8, (19)], note that the formula contains the redundant factor $1 /(n-1))$ gives

$$
r^{*}=b \frac{\|\boldsymbol{a}\|}{\sum_{i=1}^{n}\|\boldsymbol{a}[i]\| a_{i}} .
$$

Furthermore, we know that for $1 \leq j \leq n$, the vector $\boldsymbol{c}^{*}-r^{*} \boldsymbol{u}_{j}$ has to lie in the facet corresponding to $\boldsymbol{u}_{j}$. Hence the $j$ th coordinate of $\boldsymbol{c}^{*}-r^{*} \boldsymbol{u}_{j}$ has to be zero and so we find

$$
c_{j}^{*}=r^{*} \frac{\|\boldsymbol{a}\|}{\|\boldsymbol{a}[j]\|}\left(1-\frac{a_{j}^{2}}{\|\boldsymbol{a}\|^{2}}\right)=b \frac{\|\boldsymbol{a}[j]\|}{\sum_{i=1}^{n}\|\boldsymbol{a}[i]\| a_{i}} .
$$

The numbers $c_{j}^{*}$ are in general not rational. However we can find in polynomial time a rational approximation $\boldsymbol{c}$ of the vector $\boldsymbol{c}^{*}$ which satisfies the statement of the lemma.

Suppose that

$$
b \geq(1+\delta) \mu(1, n) \sum_{i=1}^{n}\|\boldsymbol{a}[i]\| a_{i},
$$

where $\mu(m, n)=2^{(n-m) / 2-1} p(m, n)$, as in Section 5.1, To prove Theorem 1.3 we have to find in polynomial time an integer point in $P\left(\boldsymbol{a}^{T}, b\right)$. 
Recall that $\boldsymbol{a}[i]=\left(a_{1}, \ldots, a_{i-1}, a_{i+1}, \ldots, a_{N}\right)$. We propose the following modification of the algorithm from Section 5 for solving this problem.

Steps 1, 3 and 4 remain the same. Step 2 will be modified as follows

Step $2^{*}$ : Find a point $\boldsymbol{c}$ such that $P\left(\boldsymbol{a}^{T}, b\right)$ contains an $(n-m)$-dimensional ball centered at $c$ and of radius

$$
r>\frac{b\|\boldsymbol{a}\|}{(1+\delta) \sum_{i=1}^{n}\|\boldsymbol{a}[i]\| a_{i}} .
$$

Step $2^{*}$ is justified by Lemma 6.1. To prove correctness of the algorithm, it is now enough to show that the point $\boldsymbol{z}$ obtained at Step 4 satisfies

$$
\|z-c\| \leq r .
$$

Observe that, as in the proof of Theorem 1.1, the inequality (5.5) holds. Consequently, by Lemma 3.3 and (6.2) we obtain the inequality (6.3). Thus the condition (1.4) can be replaced by (1.8).

Next, let us replace $\mu(m, n)=2^{(n-m) / 2-1} p(m, n)$ by $\mu(m, n)=p(m, n)$. In this case, by Theorem 1.4 (for simplicity applied with $\rho_{k} /(\operatorname{det}(L))^{2}$ replaced by 1) we obtain the inequality (6.3) as well. Thus the condition (1.5) can be replaced by (1.9).

\section{ACKnowledgement}

The authors are very grateful to anonymous referees for numerous comments and remarks which significantly enhanced the exposition and improved results of this paper and, especially, for suggesting a new proof of Theorem 1.4 .

\section{REFERENCES}

[1] K. Aardal, A. Lenstra, Hard equality constrained integer knapsacks, Math. Oper. Res. 29 (2004), no. 3, 724-738.

[2] I. Aliev and M. Henk, Feasibility of integer knapsacks, SIAM J. Optimization, 20 (2010), 2978-2993.

[3] I. Aliev and M. Henk, Integer knapsacks: average behavior of the Frobenius numbers, Mathematics of Operations Research, Mathematics of Operations Research 34 (3), 2009, 698-705.

[4] L. Babai, On Lovsz' lattice reduction and the nearest lattice point problem, Combinatorica 6 (1986), no. 1, 1-13.

[5] D. Beihoffer, J. Hendry, A. Nijenhuis, S. Wagon, Faster algorithms for Frobenius numbers, Electron. J. Combin. 12 (2005), Research Paper 27, 38 pp. (electronic).

[6] J. W. S. Cassels, An introduction to the Geometry of Numbers, Springer-Verlag 1971.

[7] F. Eisenbrand, G. Shmonin, Parametric integer programming in fixed dimension, Math. Oper. Res. 33 (2008), no. 4, 839-850.

[8] L. Fukshansky, S. Robins, Frobenius problem and the covering radius of a lattice, Discrete Comput. Geom. 37 (2007), no. 3, 471-483.

[9] M. Grötschel, L. Lovász, A. Schrijver, Geometric algorithms and combinatorial optimization, Algorithms and Combinatorics: Study and Research Texts, 2. SpringerVerlag, Berlin, 1988.

[10] P. M. Gruber, Convex and discrete geometry, Springer, Berlin, 2007. 
[11] P. M. Gruber, C. G. Lekkerkerker, Geometry of Numbers, North-Holland, Amsterdam 1987.

[12] P. Hansen, J. Ryan, Testing integer knapsacks for feasibility, European Journal of Operational Research, 88, 1996, no. 3, 578-582.

[13] R. Kannan, Lattice translates of a polytope and the Frobenius problem, Combinatorica, 12(2)(1992), 161-177.

[14] R. M. Karp, Reducibility among combinatorial problems, in Complexity of Computer Computations, R. E. Miller and J. W. Thatcher, Eds, Plenum, New York, 1972, 85-103.

[15] M. J. Knight, A generalization of a result of Sylvester's, J. Number Theory 12 (1980), no. 3, 364-366.

[16] J. Lee, S. Onn, R. Weismantel, Nonlinear optimization over a weighted independence system, submitted.

[17] A. K. Lenstra, H. W. Lenstra Jr., L. Lovsz, Factoring polynomials with rational coefficients, Math. Ann. 261 (1982), no. 4, 515-534.

[18] J. Martinet, Perfect lattices in Euclidean spaces, Grundlehren der Mathematischen Wissenschaften, vol. 327 (2003), Springer-Verlag, Berlin.

[19] P. McMullen, Determinants of lattices induced by rational subspaces, Bull. London Math. Soc. 16 (1984), no. 3, 275-277.

[20] C. H. Papadimitriou, K. Steiglitz, Combinatorial optimization: algorithms and complexity, Dover Publications, Inc., Mineola, NY, 1998.

[21] P. Pleasants, H. Ray, J. Simpson, The Frobenius problem on lattices, Australas. J. Combin. 32 (2005), 27-45.

[22] J. L. Ramírez Alfonsín, The Diophantine Frobenius problem, Oxford Lecture Series in Mathematics and Its Applications, 2005.

[23] J. L. Ramírez Alfonsín, Complexity of the Frobenius problem, Combinatorica, 16 (1996), no. 1, 143-147.

[24] W. M. Schmidt, The distribution of sublattices of $Z^{m}$, Monatsh. Math. 125 (1998), no. $1,37-81$.

[25] A. Schrijver, Theory of linear and integer programming, Wiley, Chichester, 1986.

[26] R. J. Simpson, R. Tijdeman, Multi-dimensional versions of a theorem of Fine and Wilf and a formula of Sylvester, Proc. Amer. Math. Soc. 131 (2003), no. 6, 1661-1671.

[27] J. Vaaler, A geometric inequality with applications to linear forms, Pacific J. Math. 83 (1979), no. 2, 543-553.

School of Mathematics and Wales Institute of Mathematical and Computational Sciences, Cardiff University, Senghennydd Road, Cardiff, Wales, UK

E-mail address: alievi@cf.ac.uk

FAKultät FÜr Mathematik, Otto-Von-Guericke Universität MagdeburG, UniVERsitätsplatz 2, D-39106 Magdeburg, Germany

E-mail address: martin.henk@ovgu.de 